\title{
Facile Synthesis of Cooperative Acid-Base Catalysts by Clicking Cysteine and Cysteamine on an Ethylene-Bridged Periodic Mesoporous Organosilica
}

\author{
Judith Ouwehand, ${ }^{[a]}$ Jeroen Lauwaert, ${ }^{[b]}$ Dolores Esquivel, ${ }^{[a]}$ Kevin Hendrickx, ${ }^{[a, c]}$ \\ Veronique Van Speybroeck, ${ }^{[c]}$ Joris W. Thybaut, ${ }^{[b]}$ and Pascal Van Der Voort ${ }^{*[a]}$
}

Abstract: A periodic mesoporous organosilica (PMO) that contains ethylene bridges was functionalized to obtain a series of cooperative acid-base catalysts. A straightforward, single-step procedure was devised to immobilize cysteine and cysteamine on the support material by means of a photoinitiated thiol-ene click reaction. Likewise, PMO materials capped with hexamethyldisilazane (HMDS) were used to support both compounds. This resulted in different materials in which the amine site was promoted by carboxylic acid groups, surface silanol groups, or both. The catalysts were tested in the aldol reaction of 4-nitrobenzaldehyde and acetone. It was found that silanol groups have a stronger promoting effect on the amine than the carboxylic acid group. The highest turnover frequency (TOF) was obtained for an amine-functionalized material that contained only silanol promoting sites. The loading of the active sites also had a significant effect on the activity of the catalysts, which was rationalized on the basis of a computational study.

\section{Introduction}

The incorporation of antagonistic acid and base groups on mesoporous materials for cooperative catalysis has been an important research topic in recent years. This type of material is especially useful for carbon-carbon coupling reactions such as aldol, ${ }^{[1]}$ nitroaldol, ${ }^{[1 c, 2]}$ and Knoevenagel reactions. ${ }^{[3]}$ These coupling reactions are catalyzed by an amine, but the presence of an acid function can promote the activity. The literature holds examples of how several different types of acid and amine groups were incorporated onto silica gel or ordered mesoporous silicas with the formation of cooperative acid-base catalysts. The incorporation of the acid and base groups usually occurs through grafting or co-condensation, but these synthesis methods often require several reaction steps including the protection and deprotection of the amine or an oxidation reaction. ${ }^{[1 f, 1 h, 3,4]}$ Alternatively, the antagonistic groups have been incorporated by means of successive addition of the organosilanes during a co-condensation synthesis ${ }^{[1 a-1 c]}$ or by means of their successive grafting. ${ }^{[1 e]}$

Periodic mesoporous organosilicas (PMOs) are materials derived from a bridged silane of the form $\left(\mathrm{R}^{\prime} \mathrm{O}\right)_{3} \mathrm{Si}-\mathrm{R}-\mathrm{Si}\left(O \mathrm{R}^{\prime}\right)_{3}$ in which $\mathrm{R}$ represents a functional organic bridge. The functional

[a] Department of Inorganic and Physical Chemistry, Ghent University Krijgslaan 281-S3, 9000 Ghent, Belgium

E-mail: pascal.vandervoort@ugent.be www.comoc.ugent.be

[b] Department of Chemical Technology, Ghent University Technologiepark 914, 9052 Ghent, Belgium

[c] Center for Molecular Modeling, Ghent University Technologiepark 903, 9052 Zwijnaarde, Belgium

Supporting information and $O R C I D(s)$ from the author(s) for this article are available on the WWW under http://dx.doi.org/10.1002/ejic.201501179. groups contained in PMO materials have previously been exploited to incorporate spatially separated amino and sulfonic acid groups. However, these approaches make use of several synthetic steps, including protection and deprotection steps or epoxidation and amination steps. ${ }^{[2,5]}$ In this work, we took advantage of ethylene functionalities embedded in a PMO material to incorporate carboxylic acid and amine groups by means of a single thiol-ene click reaction using the amino acid cysteine. A similar approach was employed for the incorporation of the sole amine functionality through the grafting of cysteamine. In this type of click reaction, a thiol and an ethylene moiety are linked to form a thioether bond. ${ }^{[6]}$ Thus, in this case, the catalytically active sites are not linked to the carrier material through siloxane bonds, as is the case when classical grafting or co-condensation procedures are used, ${ }^{[1 f]}$ but through hydrothermally more stable thioether bonds. In this way, the thiolene click reaction provides a reliable procedure for a straightforward, single-step grafting method.

In this work, the catalytic activity of the functionalized PMO materials in the aldol reaction of 4-nitrobenzaldehyde and acetone was investigated. The acid promoting properties of the carboxylic acid groups were compared to that of the silanol groups, which are intrinsically present on the PMO surface. Subsequently, the influence of the loading of the catalytic center was investigated, as well as the potential of inducing chirality in the reaction products.

\section{Results and Discussion}

\section{Synthesis of the Materials}

Ethylene-bridged PMO materials were prepared by means of the condensation of (E)-1,2-bis(triethoxysilyl)ethylene (BTEE) un- 


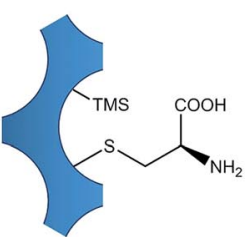

Cys-ePMO-HMDS

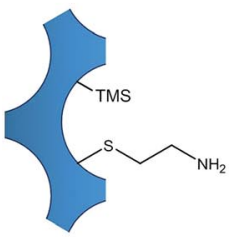

Amine-ePMO-HMDS

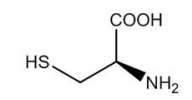

Cysteine

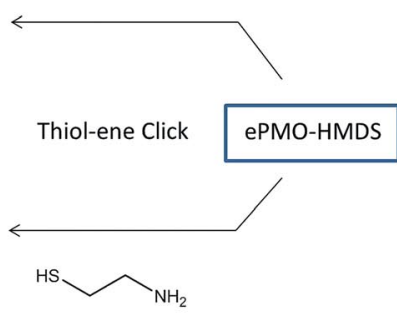

Cysteamine

Scheme 1. Overview of the catalyst functionalization (TMS = trimethylsilyl).

der acidic conditions by using P123 as template and butanol as additive. The double bonds of the obtained ethylene PMO (ePMO) were used as an anchoring point for the grafting of cysteine and cysteamine by means of a photoinitiated thiol-ene click reaction to result in Cys-ePMO and Amine-ePMO samples, respectively (Scheme 1). The reaction time of the thiol-ene click reaction was varied to obtain a series of these materials with different loadings of the active sites. A second type of catalyst was synthesized by treating the ePMO with hexamethyldisilazane (HMDS) to cap the surface silanol groups. Subsequently, the capped samples were also grafted with cysteine and cysteamine. These samples are indicated with the suffix -HMDS.

Through this approach, catalysts that contained a primary amine combined with carboxylic acid groups, surface silanol groups, or a combination of both were obtained.

\section{Characterization of the Materials}

The structure and functionalities of the PMO materials were analyzed by means of diffuse reflectance infrared Fourier transform spectroscopy (DRIFTS), nitrogen sorption, X-ray diffraction (XRD), and elemental analysis.

The presence of the functional groups was confirmed by DRIFTS measurements. As shown in Figure 1, all non-capped samples show characteristic silanol peaks around $3720 \mathrm{~cm}^{-1}$. The peaks at $2950 \mathrm{~cm}^{-1}$ represent the $\mathrm{C}-\mathrm{H}$ stretching from the ethylene bridge and the carbon atoms in the cysteine or cysteamine groups. For ePMO-HMDS, the silanol peak is strongly reduced, thus indicating that the majority of free silanols were capped with HMDS. The increased $\mathrm{C}-\mathrm{H}$ signal at $2950 \mathrm{~cm}^{-1}$ for the same sample is due to the presence of the trimethylsilyl groups that originate from HMDS.

In the Cys-ePMO spectrum, a carbonyl signal can be observed at $1730 \mathrm{~cm}^{-1}$. In the Amine-ePMO spectrum, two peaks at 3315 and $3365 \mathrm{~cm}^{-1}$ can be assigned to $\mathrm{N}-\mathrm{H}$ stretching. In the Cys-ePMO and Amine-ePMO spectra, the signal at $1615 \mathrm{~cm}^{-1}$ is due to $\mathrm{N}-\mathrm{H}$ bending.

After grafting cysteine and cysteamine, the loading of the functional groups was determined by elemental analysis of nitrogen (Table 1). For Cys-ePMO and Amine-ePMO, catalysts of
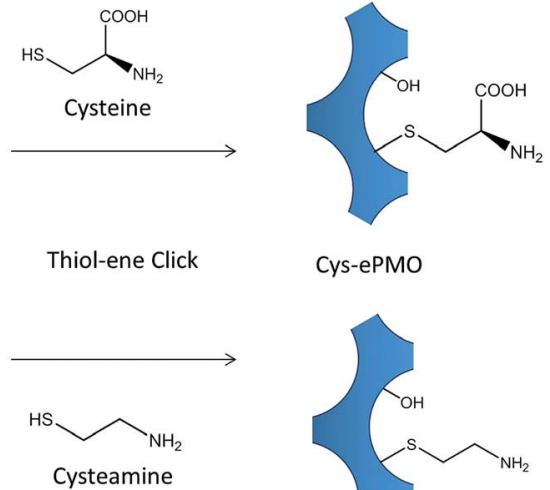

Cys-ePMO

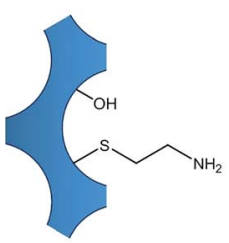

Amine-ePMO

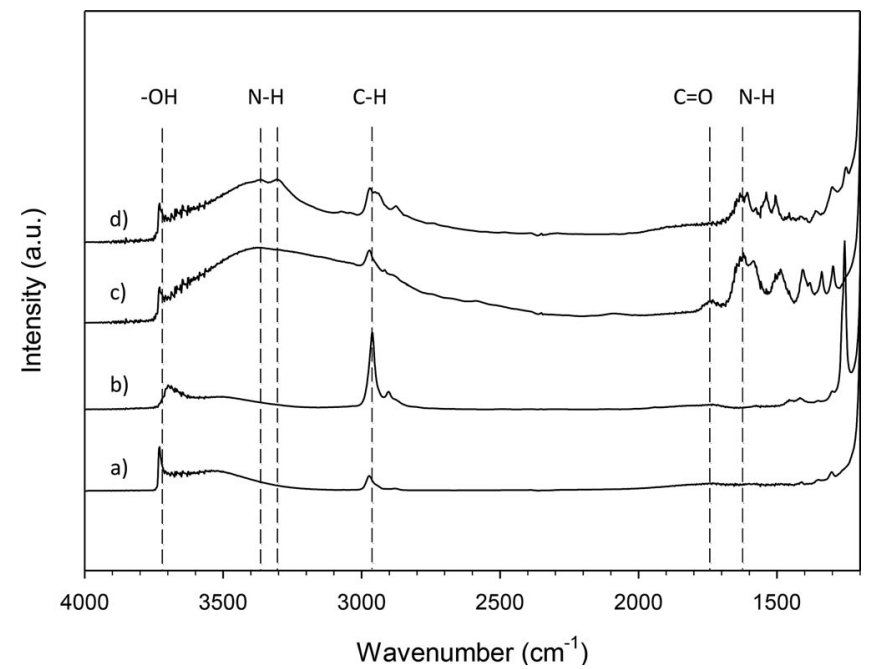

Figure 1. DRIFTS spectra of the materials: (a) ePMO, (b) ePMO-HMDS, (c) CysePMO, and (d) Amine-ePMO.

different loadings were obtained by varying the reaction time of the thiol-ene click reaction. Thus, the loadings of the catalysts range from 0.08 to $0.84 \mathrm{mmol} \mathrm{g}^{-1}$ of nitrogen.

The nitrogen-sorption isotherms are shown in Figure 2. All materials exhibit type IV isotherms and $\mathrm{H} 1$ type hysteresis loops at relative pressures in the range of $0.4-0.8$, indicative of cylindrical mesopores. The specific BET surface area $\left(S_{\mathrm{BET}}\right)$, average pore size $\left(d_{\mathrm{p}}\right)$, pore volume $\left(V_{\mathrm{p}}\right)$, and wall thickness are summarized in Table 1. The starting ePMO and ePMO-HMDS materials have large surface areas of 890 and $751 \mathrm{~m}^{2} \mathrm{~g}^{-1}$, respectively. After functionalization with cysteine and cysteamine, the specific surface area of the samples decreases, which is due to an effective decrease in surface area as well as to an increase in weight after the grafting procedure. All samples have pore diameters around $5 \mathrm{~nm}$ and a wall thickness between 4 and $6 \mathrm{~nm}$. This large wall thickness provides stability to the PMO materials.

The XRD patterns of the materials before and after grafting of cysteine and cysteamine are shown in Figure 3. All the materials show the characteristic (100), (110), and (200) reflections of a hexagonal pore ordering, which confirms that after func- 
Table 1. Structural properties and functional-group loadings of the PMO materials.

\begin{tabular}{|c|c|c|c|c|c|c|}
\hline Sample & $S_{B E T}{ }^{[a]}\left[\left(m^{2} g^{-1}\right]\right.$ & $d_{\mathrm{p}}^{[\mathrm{b}]}[\mathrm{nm}]$ & $V_{\mathrm{p}}^{[c]}\left[\mathrm{mL} \mathrm{\textrm {g } ^ { - 1 } ]}\right.$ & $a_{0}^{[\mathrm{d}]}[\mathrm{nm}]$ & Wall thickness ${ }^{[\mathrm{e}]}[\mathrm{nm}]$ & Loading $^{[f]}\left[\mathrm{mmol}^{\mathrm{g}-1}\right]$ \\
\hline ePMO & 890 & 5.2 & 0.98 & 9.4 & 4.2 & - \\
\hline ePMO-HMDS & 751 & 5.4 & 0.85 & 10.6 & 5.2 & - \\
\hline Cys-ePMO-1 & 610 & 5.4 & 0.71 & 10.2 & 4.8 & 0.08 \\
\hline Cys-ePMO-2 & 576 & 5.5 & 0.80 & 10.2 & 4.7 & 0.41 \\
\hline Cys-ePMO-3 & 496 & 5.0 & 0.39 & 10.6 & 5.6 & 0.84 \\
\hline Cys-ePMO-HMDS & 604 & 5.0 & 0.83 & 9.8 & 4.8 & 0.15 \\
\hline Amine-ePMO-1 & 518 & 4.8 & 0.62 & 9.1 & 4.3 & 0.49 \\
\hline Amine-ePMO-2 & 373 & 5.5 & 0.63 & 10.1 & 4.6 & 0.65 \\
\hline Amine-ePMO-HMDS & 633 & 5.3 & 0.861 & 10.8 & 5.5 & 0.46 \\
\hline
\end{tabular}

[a] Specific Brunauer-Emmett-Teller surface area. [b] Barrett-Joyner-Halenda pore diameter determined from desorption isotherm. [c] Pore volume determined from desorption isotherm. [d] Lattice parameter calculated by $a_{0}=2 d_{100} / \sqrt{ } 3$. [e] Calculated as the difference between $a_{0}$ and $d_{\mathrm{p}}$. [f] Loading of nitrogen from elemental analysis.
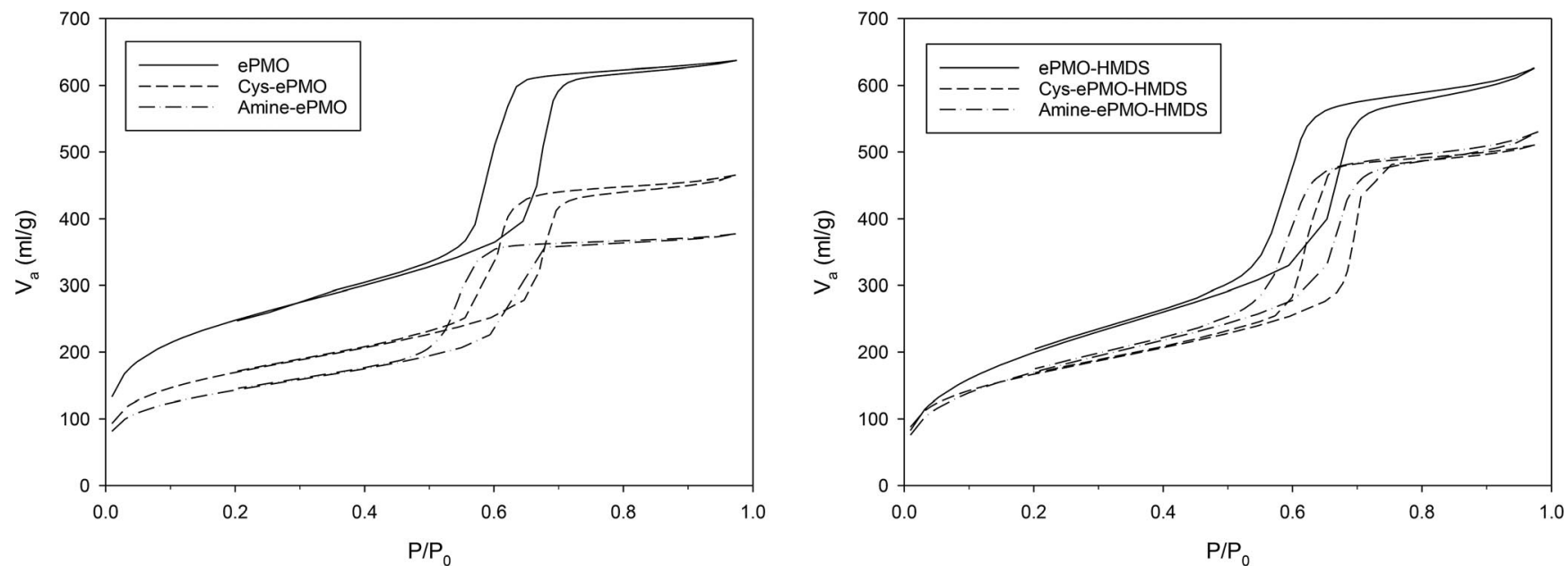

Figure 2. Nitrogen-sorption isotherms of the non-capped (left) and capped (right) samples.
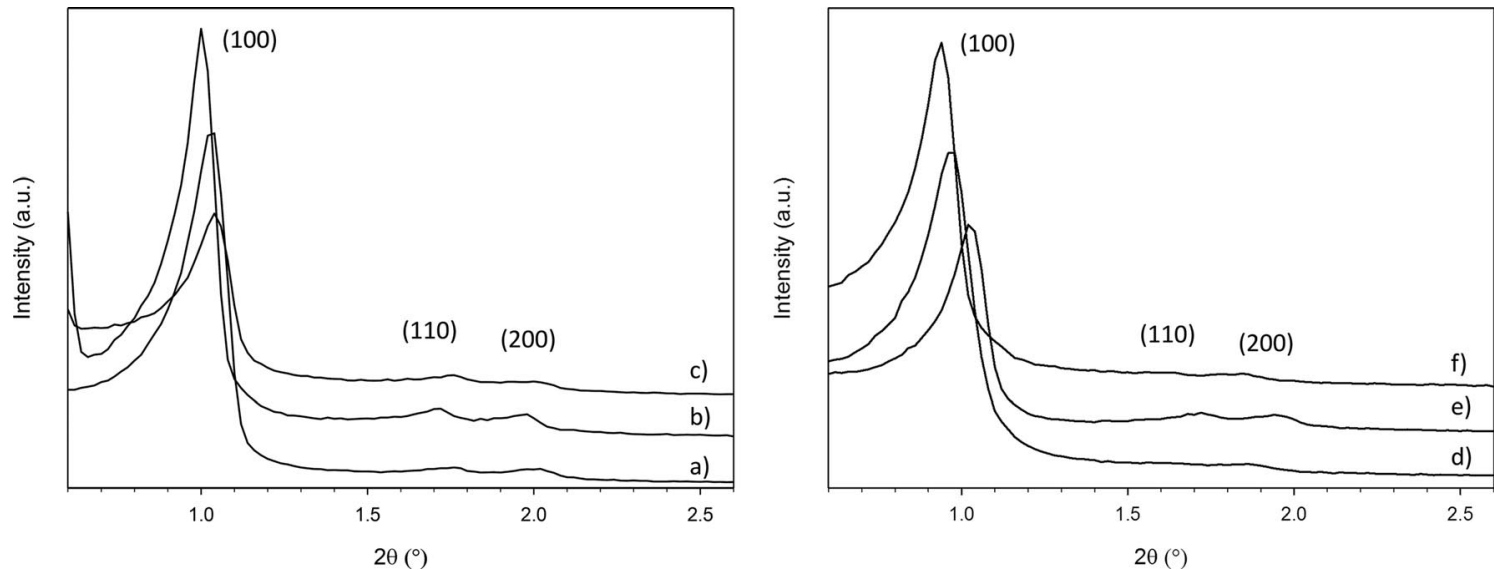

Figure 3. XRD patterns of the non-capped (left) and capped (right) samples: (a) ePMO, (b) Cys-ePMO, (c) Amine-ePMO, (d) ePMO-HMDS, (e) Cys-ePMO-HMDS, and (f) Amine-ePMO-HMDS.

tionalization, all samples retain their ordered pore structure of the $P 6 \mathrm{~mm}$ point group.

\section{Catalytic Results}

\section{Activity and Selectivity}

The catalytic activities of the functionalized materials were tested in the aldol reaction of 4-nitrobenzaldehyde and acetone
(Figure 4). In each experiment, the amount of catalyst added to the reaction mixture was adjusted such that the desired amount of amines was present (i.e., 4 mol-\% with respect to the 4-nitrobenzaldehyde concentration) to be able to directly compare the activity of the different materials to each other.

The conversion increased linearly with the reaction time up to a conversion of approximately $95 \%$, thus indicating that the reaction rate remained constant and that the materials were 
<smiles></smiles>

Figure 4. Aldol condensation of 4-nitrobenzaldehyde and acetone with the formation of aldol products $\mathbf{1}$ and $\mathbf{2}$.

Table 2. Catalytic data of the functionalized PMO materials.

\begin{tabular}{|c|c|c|c|c|}
\hline Sample & Loading $^{[\mathrm{a}]}\left[\mathrm{mmol} \mathrm{g}^{-1}\right]$ & TOF $\times 10^{-4}\left[\mathrm{~s}^{-1}\right]$ & Selectivity ${ }^{[b]}[\%]$ & $e e^{[c]}[\%]$ \\
\hline Cys-ePMO-1 & 0.08 & 5.5 & 92.6 & $<5$ \\
\hline Cys-ePMO-2 & 0.41 & 2.5 & 95.0 & $<5$ \\
\hline Cys-ePMO-3 & 0.84 & 1.1 & 96.8 & $<5$ \\
\hline Cys-ePMO-HMDS & 0.15 & 0.9 & 95.3 & $25( \pm 5)$ \\
\hline Amine-ePMO-1 & 0.49 & 3.5 & 96.5 & $<5$ \\
\hline Amine-ePMO-2 & 0.65 & 2.3 & 96.5 & $<5$ \\
\hline Amine-ePMO-HMDS & 0.46 & 0.3 & 91.2 & $<5$ \\
\hline
\end{tabular}

[a] Loading of nitrogen, from elemental analysis. [b] Towards aldol product 1 (Figure 4). [c] Towards the (R) isomer of $\mathbf{1}$.

stable during the catalytic tests. Figure 5 shows the data points at the start of the reaction, which were used to determine the turnover frequency (TOF) values of the different catalysts.

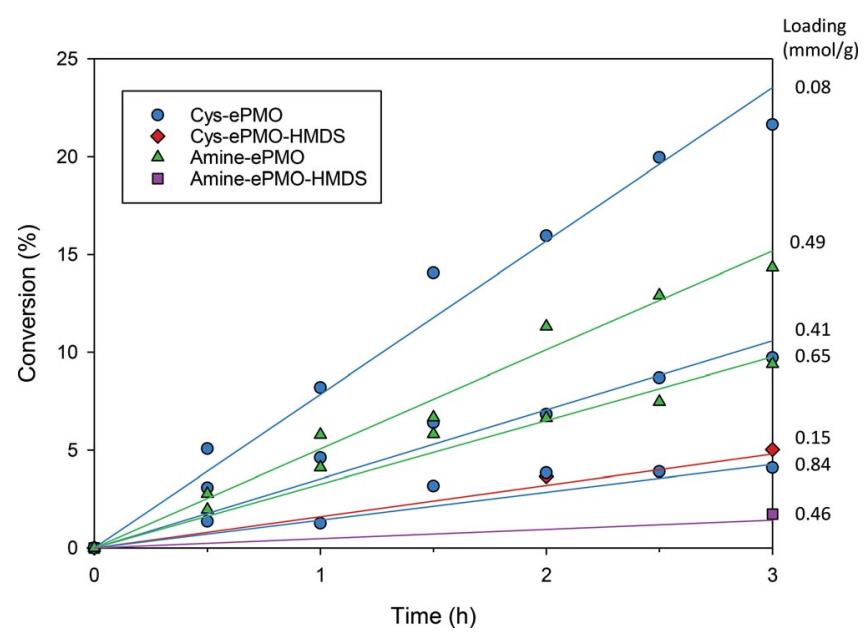

Figure 5. Data points of the conversion curves used to determine the TOF values.

The catalytic data of the catalysts are shown in Table 2. All catalysts were able to promote the reaction with a high selectivity towards aldol product 1 (>90\%) with TOF values ranging from $3.0 \times 10^{-5}$ to $5.5 \times 10^{-4} \mathrm{~s}^{-1}$.

The TOF values in Table 2 can be compared to activities of aminated porous silica materials previously reported by Lauwaert et al. $^{[1]}{ }^{[1]}$ as these were tested in the same aldol reaction under identical reaction conditions. Porous silica grafted with a primary amine showed a TOF of $7.8 \times 10^{-4} \mathrm{~s}^{-1}$, which is slightly higher than those of the Amine-ePMO samples. A bifunctional silica material grafted with both amine and carboxylic acid groups showed a TOF of $9.5 \times 10^{-5} \mathrm{~s}^{-1}$, which is slightly lower than those of the Cys-ePMO samples. Overall, the catalytic activities of the PMO material reported here are in the same order of magnitude as those of the corresponding silica materials.

A recycling study performed for Cys-ePMO-1 showed that in the second catalytic run the conversion still increased linearly with reaction time, albeit with a lower slope. The TOF in the second run amounted to $55 \%$ of the original TOF. The linear character of the conversion behavior with time indicates that stable catalytic behavior was observed in a single run. More insight into the lower slope in the second run is currently being gained by means of time-on-stream measurements in a continuous flow setup and will be reported separately.

The chirality of the aldol product was investigated by chiral HPLC. The only sample that showed significant enantiomeric excess (ee) was Cys-ePMO-HMDS. These catalytic data are interpreted in the following sections.

\section{Influence of the Acid Promoting Site}

As shown in Table 2, four different types of catalysts were obtained, which each contain a primary amine combined with different types of acid promoting sites. Cys-ePMO contains both carboxylic acid and surface silanol groups. Cys-ePMO-HMDS only contains carboxylic acid groups, whereas Amine-ePMO only contains surface silanol groups. Amine-ePMO-HMDS, however, does not contain any acid promoting sites. To compare these four types of materials while minimizing the influence of the loading of the active sites, four different samples with similar amine loadings (in the range of $0.15-0.5 \mathrm{mmol} \mathrm{g}^{-1}$ ) were selected. These samples and their TOF values are shown in Figure 6.

As could be expected, Amine-ePMO-HMDS, which has no acid promoting sites, has the lowest activity. Amine-ePMO, which contains only silanol promoting groups, is more active than Cys-ePMO-HMDS, which contains only carboxylic acid promoting groups. This suggests that, for this reaction, the silanol groups on the PMO surface are more efficient promoters to the amine group. This is also immediately apparent from the fact that both capped samples have lower activities than the noncapped samples.

This observation is in agreement with previously published studies on functionalized porous silica materials. ${ }^{[1 e, 1 f]}$ In the enamine reaction mechanism, the role of the acid promoting sites is to activate the carbonyl species. However, it was observed that the grafting of carboxylic acid groups along with amine groups on the silica surface lowered the activity of the materials 


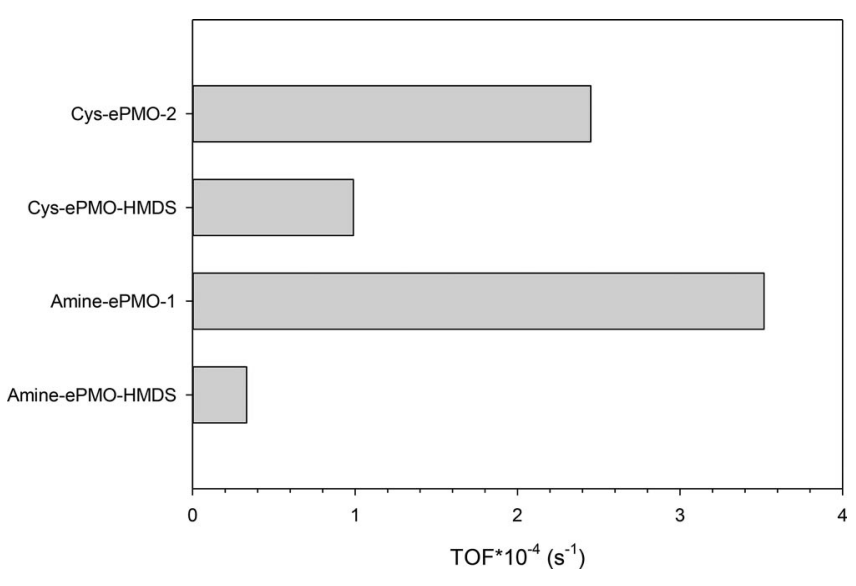

Figure 6. Catalytic activity (TOF) of the different types of catalysts.

relative to silicas functionalized with only amines. This effect was ascribed to two factors.

Firstly, it was proposed that the grafting procedure of the carboxylic acid group by means of silylation of the surface silanols effectively deactivates a fraction of the active silanols. However, this theory is not applicable to the PMO materials described here, since both functional groups were incorporated through thiol-ene click chemistry as opposed to silylation.

The second explanation for the decrease in activity is related to the relatively high acid strength of the carboxylic acid ( $\mathrm{p} K_{\mathrm{a}}$ of $1.8^{[22]}$ ) relative to the silanol groups (average $p K_{a}$ of $7.1^{[7]}$ ). This makes the carboxylic acid group a stronger hydrogen-bond donor and thus more likely to deactivate the amine. This second effect can also take place on the PMO material, with the difference that only hydrogen bonds between different cysteine moieties can occur, as the amine and carboxylic acid on a single cysteine moiety are not in a configuration that allows hydrogen bonding. The degree of hydrogen-bond formation and thus the loss of catalytic activity should then be proportional to the loading of the active sites. This effect is investigated in the following section.

The activity of Cys-ePMO, which contains both carboxylic acid and silanol groups, is lower than that of Amine-ePMO. This leads to the reasoning that a third factor should be considered, in the form of a steric hindrance effect, caused by the fact that the carboxylic acid groups are in close proximity to the amine active sites. This could make the amines less accessible to the aldol reagents, which would result in a decrease in activity of Cys-ePMO relative to Amine-ePMO.

Thus, for the PMO materials, a combination of hydrogenbond formation and steric hindrance can explain the reactivity of the different types of catalysts.

In terms of chirality of the aldol product, Cys-ePMO-HMDS was the only sample to show a significant enantioselectivity, with $25 \%$ ee. The Cys-ePMO sample does not show any significant ee. This indicates that for this sample the steric hindrance effect proposed above is taking place, as the activity lowers for Cys-ePMO relative to Amine-ePMO, but no significant ee was observed.

In conclusion, the aminated ePMO with only silanol promoters has the highest activity in the aldol condensation reaction owing to a stronger promotion by the silanol groups. This also results in a lack of enantioselectivity in all samples except CysePMO-HMDS.

\section{Influence of the Loading}

In a further catalytic investigation, the influence of the loading of the active sites was studied. For this, the Cys-ePMO and Amine-ePMO samples with different loadings were compared. The TOF values are shown in Figure 7, whereas the loadings are in Table 2. For both the cysteine- and amine-functionalized samples, the TOF increased with decreasing loading. A similar effect was reported in the literature when grafting primary amines onto silica materials with different loadings. ${ }^{[1,8]}$ The loss of activity at higher loadings was ascribed to a decrease in surrounding silanol groups. Furthermore, it was suggested that hydrogen bonds were formed between the functional groups involved, thereby causing deactivation of the amine active sites.

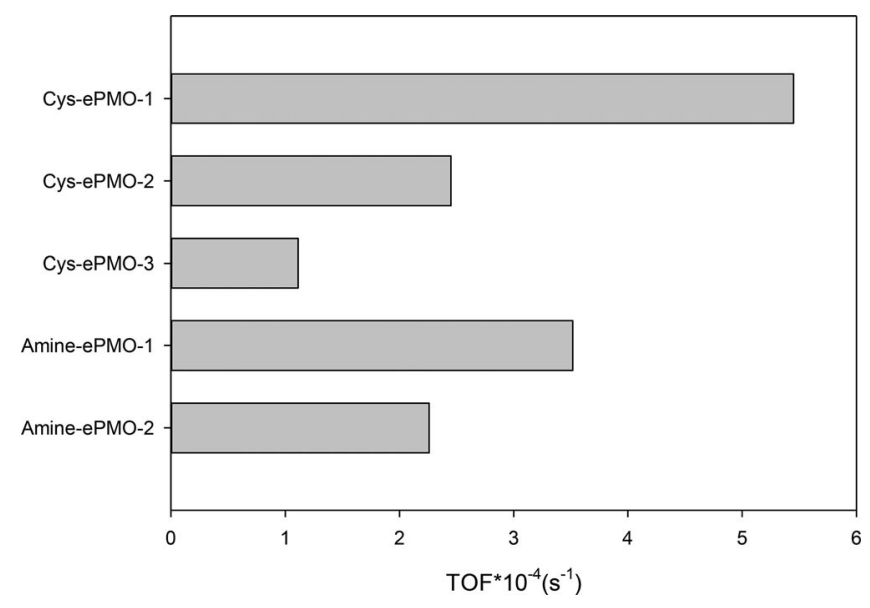

Figure 7. TOFs of the samples with different loadings.

To investigate the hydrogen-bond formation on a PMO material, a computational study based on a simplified model system was performed to obtain more insight into the interactions at the nanoscale level. Theoretical studies of PMO materials are only available in very limited examples in the literature, ${ }^{[9]}$ whereas modeling of mesoporous silicas is already more common. ${ }^{[10]}$

To investigate the possible conformations of two cysteine moieties grafted onto neighboring ethylene bridges, a simple model was used, in which the PMO backbone was kept rigid, whereas the cysteine moieties were allowed to rotate and interact. Optimizations starting from different initial geometries showed that the potential energy surface of the system has several local minima. All localized minima are shown in Figure S1 of the Supporting Information with their relative stabilities. It was observed that geometries that allow the formation of a hydrogen bond between the amine groups are about $25 \mathrm{~kJ} \mathrm{~mol}^{-1}$ more stable than the other structures.

These stable conformations were then introduced into a model in which the silanol groups and ethylene bridges were no longer static but were also allowed to rotate and interact with the cysteine moieties (Figure S2 in the Supporting Information). This resulted in the most stable conformation (struc- 
ture F), which is shown in Figure 8. This conformation is stabilized by hydrogen bonds between the amine groups, between the carboxylic acid groups, and between the amine and the silanol groups.

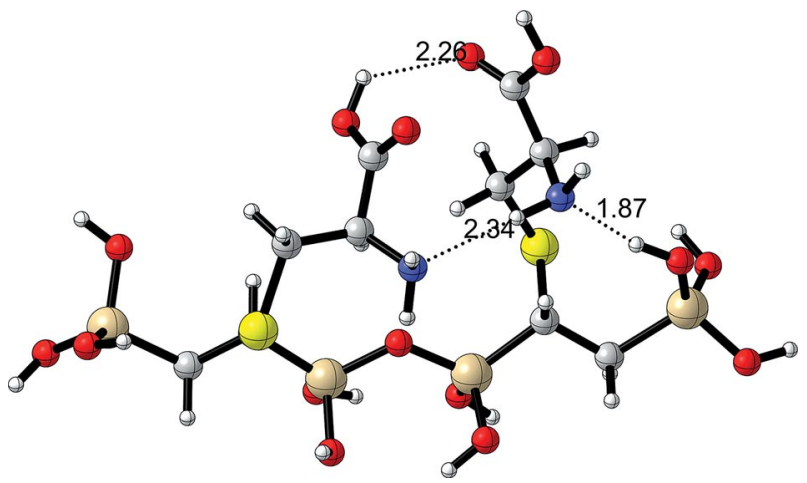

Figure 8. Most stable conformation obtained for two cysteine moieties grafted onto neighboring ethylene bridges $(\mathrm{H}=$ white, $\mathrm{C}=$ gray, $\mathrm{N}=$ blue, $\mathrm{O}=$ red, $\mathrm{Si}=$ beige, $\mathrm{S}=$ yellow).

To confirm the geometries obtained from these static calculations, first principle molecular dynamics (MD) simulations were performed. Within the timescale of the simulation (12 ps), the molecule remained mostly in this stable conformation, thus showing the strong effect of the amine hydrogen bonds. Figure 9 shows the $\mathrm{N}$...H distance during the simulation. It can be seen that the bond length stays within the range of a strong hydrogen bond, thereby confirming that this geometry is stable when allowed to rotate freely. In conclusion, the calculations confirm a large stabilizing effect owing to hydrogen-bond formation between two neighboring amine groups.

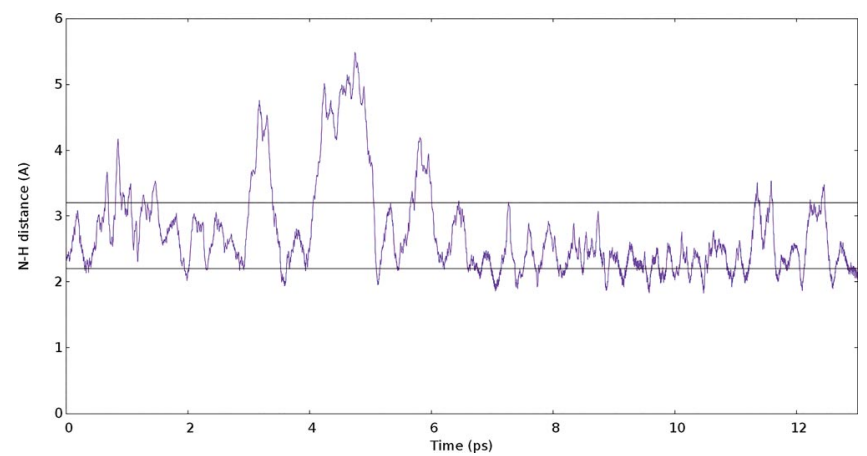

Figure 9. Evolution of the $\mathrm{N}$...H hydrogen-bond length during the MD simulation. The black lines represent the interval in which the interaction is labeled as a strong hydrogen bond. ${ }^{[11]}$

Figure 10 shows a similar model, this time of an isolated cysteine moiety grafted on the PMO backbone, representing the materials with lower loadings. In this model, the silanol groups were again allowed to rotate and interact with the cysteine group. However, in this case, no hydrogen bonding was observed.

In conclusion, these computational studies show that the configuration of two neighboring cysteine moieties is strongly stabilized by the formation of hydrogen bonds. In this case, the active amine site could be strongly inhibited in the catalytic

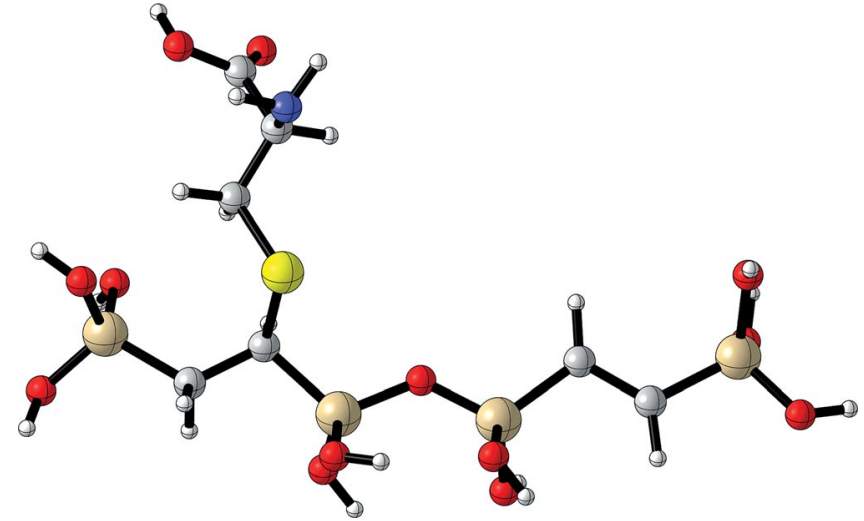

Figure 10. Geometric model of an isolated cysteine group grafted onto the PMO backbone $(\mathrm{H}=$ white, $\mathrm{C}=$ gray, $\mathrm{N}=$ blue, $\mathrm{O}=$ red, $\mathrm{Si}=$ beige, $\mathrm{S}=$ yellow).

reaction, by the change in conformation, as well as by the formed hydrogen bonds.

On the contrary, an isolated cysteine moiety is not hampered by hydrogen bonding. These findings are in accordance with the catalytic results. At high loadings, the chance of finding two neighboring cysteine sites increases, especially because hydrogen bonding can occur during the grafting procedure. The samples with higher loadings show a decrease in activity, which can be ascribed to the effects found in the computational study. The samples with the lowest loadings have the highest activities. This is in accordance with the model of the isolated cysteine site, which is more likely to occur at these lower loadings.

The Amine-ePMO samples show a similar trend in activity, which leads us to believe that a similar reasoning is valid for these aminated samples.

\section{Conclusion}

A straightforward, single-step procedure was developed to graft functional groups onto an ethylene PMO. Cysteine- and aminefunctionalized materials were obtained by a photoinitiated thiol-ene click reaction. Catalytic testing in an aldol reaction showed that the silanol groups present on the PMO surface were more effective as a promoting site to the amine than carboxylic acid groups. The catalyst without acid promoting sites showed the lowest activity. Only Cys-ePMO-HMDS showed ee, which is due to the fact that for the other catalysts the reaction was mainly promoted by the silanol groups and not by the carboxylic acid. In terms of the loading of the active sites, a lower loading gave a higher activity, which can be rationalized by the formation of hydrogen bonds between the active sites at higher loadings, as was shown in the computational study.

\section{Experimental Section}

Materials: The following chemicals were used as received: Lcysteine (>99\%, Acros), cysteamine (>95\%, TCl), 2-hydroxy-4-(2hydroxyethoxy)-2-methylpropiophenone (Irgacure 2959, $98 \%$, Aldrich), PEG-PPG-PEG Pluronic P-123 (Aldrich), 1-butanol (99.7 \%, Al- 
drich), $\mathrm{HCl}$ (37\%, Roth), vinyltriethoxysilane (97\%, $\mathrm{ABCR})$, Grubbs catalyst (first generation, $97 \%$, Aldrich), HMDS (98.5\%, ABCR), acetone (99.5\%, Aldrich), dichloromethane $\left(\mathrm{CH}_{2} \mathrm{Cl}_{2},>99.5\right.$, Roth), 4nitrobenzaldehyde ( $99 \%$, Acros), $n$-hexane (extra pure, Acros), and methyl 4-nitrobenzoate (>99\%, Aldrich).

Synthesis of ePMO and ePMO-HMDS: BTEE and the ePMO were synthesized according to previously reported procedures. ${ }^{[12]}$ The $100 \%(E)$-ethylene precursor was synthesized from vinyltriethoxysilane $(42.95 \mathrm{~mL})$, to which Grubbs catalyst $(53.5 \mathrm{mg})$ was added under an inert gas. The mixture was stirred for $1 \mathrm{~h}$ and then heated under reflux conditions at $160^{\circ} \mathrm{C}$ for $3 \mathrm{~h}$. Subsequent vacuum distillations at 90 and $160{ }^{\circ} \mathrm{C}$ were performed to obtain pure BTEE as the second distillate.

For the synthesis of the ePMO, P123 $(1 \mathrm{~g})$, butanol $(2.45 \mathrm{~mL})$, and $\mathrm{HCl}(3.42 \mathrm{~mL})$ were dissolved in water $(48.7 \mathrm{~mL})$ by stirring for $3 \mathrm{~h}$. BTEE $(1.89 \mathrm{~mL})$ was added dropwise, and the mixture was stirred at $35{ }^{\circ} \mathrm{C}$ for $5 \mathrm{~h}$. Butanol $(18 \mathrm{~mL}$ ) was added, and the material was aged at $90{ }^{\circ} \mathrm{C}$ under static conditions for $16 \mathrm{~h}$. The obtained white solid was filtered, and the template was removed by Soxhlet extraction with acetone for $6 \mathrm{~h}$. The PMO was dried at $120^{\circ} \mathrm{C}$ under vacuum.

For the synthesis of ePMO-HMDS, ePMO $(0.3 \mathrm{~g})$ was stirred in HMDS $(20 \mathrm{~mL})$ at room temperature for $5 \mathrm{~h}$. The solid was filtered and washed with $\mathrm{CH}_{2} \mathrm{Cl}_{2}$. It was then stirred in $\mathrm{CH}_{2} \mathrm{Cl}_{2}$ overnight, filtered, and dried under vacuum at $120^{\circ} \mathrm{C}$.

Grafting of Cysteine and Cysteamine: The solid was first dried at $120{ }^{\circ} \mathrm{C}$ under vacuum overnight. L-Cysteine $(0.875 \mathrm{~g})$ or cysteamine $(0.55 \mathrm{~g})$ and Irgacure $2959(0.425 \mathrm{~g})$ were dissolved in water $(50 \mathrm{~mL})$, and the solution was purged with argon. ePMO (0.425 g) or ePMOHMDS $(0.5 \mathrm{~g})$ was added, and the suspension was exposed to UV irradiation while stirring. Different loadings of cysteine or amine were obtained by varying the reaction times between $15 \mathrm{~min}$ and $24 \mathrm{~h}$.

UV reactions were performed in a Metalight Classic UV reactor equipped with 12 UV sources $(360 \mathrm{~nm}$ ) and a stirring system.

For Cys-ePMO and Cys-ePMO-HMDS, the obtained yellow solid was filtered, washed with warm water and acetone, heated to reflux in water overnight, filtered, and again washed with warm water and acetone. Finally, the product was dried under vacuum at $120^{\circ} \mathrm{C}$.

For Amine-ePMO and Amine-ePMO-HMDS, the obtained yellow solid was washed by Soxhlet extraction with acetone for $6 \mathrm{~h}$ and dried under vacuum at $120^{\circ} \mathrm{C}$.

Catalyst Characterization: FT-IR measurements were performed with a Nicolet 6700 (Thermo Scientific) with a $\mathrm{KBr}$ matrix, at $120^{\circ} \mathrm{C}$ under vacuum. Elemental analysis was performed with a Thermo Flash 2000 Thermal Analyzer (Thermo Scientific). XRD was performed with an ARL X'TRA (Thermo Scientific). Nitrogen-sorption measurements were performed with a TriStar 3000 analyzer (Micromeritics) after drying at $120^{\circ} \mathrm{C}$ under vacuum.

Catalytic Testing Procedure: The experiments were performed in a $25 \mathrm{~mL}$ two-necked round-bottomed flask equipped with a condenser and a septum. First, the supported catalyst was added to the flask so that the desired amount of amines was present (i.e., 4 mol-\% with respect to the 4-nitrobenzaldehyde concentration). The reaction mixture was prepared separately by mixing the desired amounts of acetone (50 vol.-\%), 4-nitrobenzaldehyde (0.03 mmol mL $\mathrm{m}^{-1}$ ), methyl 4-nitrobenzoate (internal standard, $0.022 \mathrm{mmol} \mathrm{mL}^{-1}$ ), and $n$-hexane (solvent, 50 vol.-\%). Afterwards, $5 \mathrm{~mL}$ of the reaction mixture was injected into the flask that contained the catalyst, and the flask was immediately placed in an oil bath at $45^{\circ} \mathrm{C}$. The reaction was monitored by taking samples (about $50 \mu \mathrm{L}$ ) of the reaction mixture. Approximately $0.5 \mathrm{~mL}$ of acetone was used to wash the syringe needle and to transfer the sample to a vial. Subsequently, the catalyst was separated from the sample by means of centrifugation. Finally, the samples were analyzed with a reversed-phase HPLC apparatus (Agilent 1100 series) equipped with a UV detector with variable wavelength. The HPLC apparatus was operated at a column temperature of $30{ }^{\circ} \mathrm{C}$ by using a gradient method with water $(0.1 \%$ trifluoroacetic acid) and acetonitrile (HPLC grade) as solvents. In this gradient method the volumetric percentage of acetonitrile was varied from 30 to $62 \%$ over a period of $7 \mathrm{~min}$. The chirality of the aldol products was analyzed by chiral HPLC (Chiralpak AS-H; hexane/ethanol, 90:10).

For the recycling experiment, the catalyst was removed by filtration after the first run, washed in $n$-hexane over $3 \mathrm{~h}$, filtered again, dried at $120^{\circ} \mathrm{C}$ under vacuum and reused in a second catalytic run under identical reaction conditions.

Computational Methodology: Static DFT calculations were carried out within the Gaussian 09 ${ }^{[13]}$ package using the hybrid B3LYP ${ }^{[14]}$ functional combined with a triple- $\zeta$ Pople basis set 6$311++G(d, p)^{[15]}$ for both optimization and frequency calculations. Empirical dispersion corrections were added by using Grimme's D3 version with Becke-Johnson damping as implemented in Gaussian. ${ }^{[16]}$ A simple model was constructed of a short chain that consisted of two linked precursor units, representative of the PMO backbone modified with one or two cysteine groups. The total stoichiometry of the model is hence $\left[\mathrm{Si}(\mathrm{OH})_{3} \mathrm{C}_{2} \mathrm{H}_{3}(\mathrm{Cyst}) \mathrm{Si}(\mathrm{OH})_{2}\right]_{2} \mathrm{O}$. The chain was first optimized without the cysteine groups, and its geometry was kept fixed during optimization of the complete model system, thus accounting for the rigidity of the PMO material. Molecular dynamics simulations were performed by using the $\mathrm{CP} 2 \mathrm{~K}^{[17]}$ package. A $22.0 \times 22.0 \times 22.0 \AA^{3}$ box subjected to periodic boundary conditions was used. The BLYP functional was used together with empirical dispersion corrections (DFT-D3) to describe the long-distance van der Waals interactions. ${ }^{[16]}$ A Gaussian and plane-waves method ${ }^{[18]}$ was chosen for all calculations by using a DZVP/TZVP Gaussian basis set for $\mathrm{Si} / \mathrm{C} / \mathrm{S} / \mathrm{H}$ and $\mathrm{O} / \mathrm{N}$, respectively, and a plane-wave basis set with a 280 Ry cutoff using GoedeckerTeter-Hutter pseudopotentials. ${ }^{[19]}$ After initial heating by using a CSVR thermostat, ${ }^{[20]}$ a Nosé-Hoover thermostat ${ }^{[21]}$ of five coupled heat baths was used to keep the system at $300 \mathrm{~K}$. In total, the NVT simulations sampled 12 ps by using a 0.5 fs time step.

Supporting Information (see footnote on the first page of this article): Additional data concerning the computational study.

\section{Acknowledgments}

This research was funded by an FWO (Fund for Scientific Research Flanders) grant with number G006813N. The work was also supported by the Long Term Structural Methusalem Funding by the Flemish Government. K. H., V. V. S., and P. V. D. V. thank the research Board of Ghent University [BOF (Special Research Fund)], and UGent GOA (Concerted Research Actions) (grant 01G00710) for financial support. The authors are grateful to Tom Planckaert for the elemental analysis measurements and Jan Goeman for the chiral HPLC analyses.

Keywords: Heterogeneous catalysis - Click chemistry · Aldol reactions . Mesoporous materials - Amino acids 
[1] a) R. Zeidan, M. Davis, J. Catal. 2007, 247, 379-382; b) N. Solin, L. Han, S. Che, O. Terasaki, Catal. Commun. 2009, 10, 1386-1389; c) S. Shylesh, A. Wagner, A. Seifert, S. Ernst, W. R. Thiel, Chem. Eur. J. 2009, 15, 70527062; d) R. K. Zeidan, S. J. Hwang, M. E. Davis, Angew. Chem. Int. Ed. 2006, 45, 6332-6335; Angew. Chem. 2006, 118, 6480; e) J. Lauwaert, E. G. Moschetta, P. Van Der Voort, J. W. Thybaut, C. W. Jones, G. B. Marin, J. Catal. 2015, 325, 19-25; f) N. A. Brunelli, K. Venkatasubbaiah, C. W. Jones, Chem. Mater. 2012, 24, 2433-2442; g) Y. Shao, J. Guan, S. Wu, H. Liu, B. Liu, Q. Kan, Microporous Mesoporous Mater. 2010, 128, 120-125; h) X. Yu, Y. Zou, S. Wu, H. Liu, J. Guan, Q. Kan, Mater. Res. Bull. 2011, 46, 951-957; i) J. Lauwaert, E. De Canck, D. Esquivel, J. W. Thybaut, P. Van Der Voort, G. B. Marin, ChemCatChem 2014, 6, 255-264; j) J. Lauwaert, E. De Canck, D. Esquivel, P. Van Der Voort, J. W. Thybaut, G. B. Marin, Catal. Today 2015, 246, 35-45; k) X. Yu, X. Yu, S. Wu, B. Liu, H. Liu, J. Guan, Q. Kan, J. Solid State Chem. 2011, 184, 289-295.

[2] a) M. Sasidharan, S. Fujita, M. Ohashi, Y. Goto, K. Nakashima, S. Inagaki, Chem. Commun. 2011, 47, 10422-10424; b) S. Shylesh, A. Wagener, A. Seifert, S. Ernst, W. R. Thiel, Angew. Chem. Int. Ed. 2010, 49, 184-187; Angew. Chem. 2010, 122, 188.

[3] F. Shang, J. Sun, H. Liu, C. Wang, J. Guan, Q. Kan, Mater. Res. Bull. 2012, 47, 801-806.

[4] Y. Shao, H. Liu, X. Yu, J. Guan, Q. Kan, Mater. Res. Bull. 2012, 47, 768-773.

[5] A. M. J. Alauzun, C. Reye, R. J. P. Corriu, J. Am. Chem. Soc. 2006, 128, 8718-8719.

[6] A. B. Lowe, Polym. Chem. 2010, 1, 17-36.

[7] N. A. Brunelli, C. W. Jones, J. Catal. 2013, 308, 60-72.

[8] a) J. C. Hicks, R. Dabestani, A. C. Buchanan, C. W. Jones, Chem. Mater. 2006, 18, 5022-5032; b) M. W. McKittrick, C. W. Jones, Chem. Mater. 2003, 15, 1132-1139; c) J. C. Hicks, C. W. Jones, Langmuir 2006, 22, 2676-2681.

[9] M. Sharifi, C. Köhler, P. Tölle, T. Frauenheim, M. Wark, Small 2011, 7, 10861097.

[10] A. Rimola, D. Costa, M. Sodupe, J. F. Lambert, P. Ugliengo, Chem. Rev. 2013, 113, 4216-4313.

[11] G. A. Jeffrey, An Introduction to Hydrogen Bonding, Oxford University Press, 1997

[12] C. Vercaemst, M. Ide, B. Allaert, N. Ledoux, F. Verpoort, P. Van Der Voort, Chem. Commun. 2007, 2261-2263.
[13] M. J. Frisch, G. W. Trucks, H. B. Schlegel, G. E. Scuseria, M. A. Robb, J. R. Cheeseman, G. Scalmani, V. Barone, B. Mennucci, G. A. Petersson, H. Nakatsuji, M. Caricato, X. Li, H. P. Hratchian, A. F. Izmaylov, J. Bloino, G. Zheng, J. L. Sonnenberg, M. Hada, M. Ehara, K. Toyota, R. Fukuda, J. Hasegawa, M. Ishida, T. Nakajima, Y. Honda, O. Kitao, H. Nakai, T. Vreven, J. A. Montgomery Jr., J. E. Peralta, F. Ogliaro, M. Bearpark, J. J. Heyd, E. Brothers, K. N. Kudin, V. N. Staroverov, R. Kobayashi, J. Normand, K. Raghavachari, A. Rendell, J. C. Burant, S. S. Iyengar, J. Tomasi, M. Cossi, N. Rega, J. M. Millam, M. Klene, J. E. Knox, J. B. Cross, V. Bakken, C. Adamo, J. Jaramillo, R. Gomperts, R. E. Stratmann, O. Yazyev, A. J. Austin, R. Cammi, C. Pomelli, J. W. Ochterski, R. L. Martin, K. Morokuma, V. G. Zakrzewski, G. A. Voth, P. Salvador, J. J. Dannenberg, S. Dapprich, A. D. Daniels, Ö. Farkas, J. B. Foresman, J. V. Ortiz, J. Cioslowski, D. J. Fox, Gaussian 09, revision D.01, Gaussian, Inc., Wallingford, CT, USA, 2009.

[14] a) A. D. J. Becke, J. Chem. Phys. 1993, 98, 5648-5652; b) H. Lee, W. Yang, R. G. Parr, Phys. Rev. B 1988, 37, 785-789.

[15] R. Krishnan, J. Binkley, R. Seeger, J. Pople, J. Chem. Phys. 1980, 72, 650654.

[16] a) S. Grimme, J. Anthony, S. Ehrlich, H. Krieg, J. Chem. Phys. 2010, 132, 154104; b) S. Grimme, S. Ehrlich, L. Goerigk, J. Comput. Chem. 2011, 32, 1456-1465.

[17] J. Hutter, M. Iannuzzi, F. Schiffmann, J. VandeVondele, Wiley Interdiscip. Rev.: Comput. Mol. Sci. 2014, 4, 15-25.

[18] G. Lippert, J. Hutter, M. Parrinello, Mol. Phys. 1997, 92, 477-487.

[19] a) C. Hartwigsen, S. Goedecker, J. Hutter, Phys. Rev. B 1998, 58, 36413662 ; b) S. Goedecker, M. Teter, J. Hutter, Phys. Rev. B 1996, 54, $1703-$ 1710.

[20] G. Bussi, D. Donadio, M. Parrinello, J. Chem. Phys. 2007, 126, 014101.

[21] a) S. Nosé, Mol. Phys. 2006, 52, 255-268; b) S. Nosé, J. Chem. Phys. 1984, $81,511$.

[22] J. M. Berg, J. L. Tymoczko, L. Stryer, Biochemistry, 5th ed., W. H. Freeman, New York, 2002.

Received: October 15, 2015

Published Online: January 15, 2016 